\title{
Impact of diabetic retinopathy screening on a British district population: case detection and blindness prevention in an evidence-based model
}

\author{
Max O Bachmann, Sally J Nelson
}

\begin{abstract}
Objectives-To quantify case detection and blindness prevention attainable through screening for diabetic retinopathy in a district population.

Design-Literature review including a pooled estimate of screening test sensitivity, and quantitative modelling, including sensitivity analyses.

Setting and patients-The diabetic population of a typical district health authority or health board.

Main results-Evidence suggests that in a British general practice based diabetic population, prevalence of retinopathy requiring treatment would be between $1 \%$ and $6 \%$; annual incidence of blindness among diabetics with retinopathy requiring treatment would be between $6 \%$ and $9 \%$; sensitivity of screening tests in detecting retinopathy requiring treatment would be between $50 \%$ and $88 \%$; and treatment could prevent $77 \%$ of expected cases of blindness. Of those screened, about $4 \%$ would be correctly detected as requiring treatment during an initial screening round, but this yield could decrease to about $1 \%$ in subsequent annual screening rounds. Of those treated, about $6 \%$ would be prevented from going blind within a year of treatment and $34 \%$ within 10 years of treatment.
\end{abstract}

Conclusions-Screening and early treatment of diabetic retinopathy can prevent substantial disability. The effectiveness and efficiency of screening could be enhanced by improving the performance of current tests or increasing use of mydriatic retinal photography, and by increasing uptake, particularly among diabetics at greatest risk.

(F Epidemiol Community Health 1998;52:45-52)

Department of Social Medicine, University of Bristol, Whiteladies Road, Bristol BS8 2PR M O Bachmann

Avon Health Authority, Kings Square House, Kings Square, Bristol BS2 8EE

S J Nelson

Correspondence to:

Dr Bachmann.

Accepted for publication 7 March 1997 diabetic patients in practices or localities; call and recall of patients to examinations; diagnostic examination at general practices, opticians premises, hospitals or mobile vans; examination of referred cases at ophthalmology outpatient departments and laser photocoagulation of suitable cases, with training and audit as quality assurance mechanisms. It is however not obvious from primary research that the expected health gain from an intensive screening programme makes retinopathy screening a leading priority for health authorities. There is also no consensus about which screening test is most appropriate.

No study has clearly shown a decrease in blindness incidence directly attributable to a retinopathy screening programme. No randomised controlled trial of screening has been reported, and given current knowledge it would be unethical to randomise diabetic patients to a control group that was not offered screening. Although there have been several estimates of yield from screening programmes, ${ }^{6-10}$ no studies have included comparisons with concurrent control populations, nor have any demonstrated the impact of screening in preventing blindness. To determine the importance of retinopathy screening it is necessary to synthesise various research evidence, to examine how the different elements of screening programme would interrelate, and to quantify the probable consequences.

Previous studies have modelled the potential impact of screening on blindness. Rohan and colleagues $^{11}$ estimated that if all 450000 people in England and Wales with diabetes and under 70 years of age were screened annually, then 260 cases of blindness could be prevented, or one per 1730 screened. The overview included a meta-analysis of studies of treatment effectiveness, but had several limitations. The model was based on a single incomplete study of test validity, and the blindness incidence was based on routine data from the 1960s. It did not examine the relation between blindness prevention and time since treatment, or the sensitivity of the model to the various assumptions upon which it was based. United States studies have modelled the economic consequences of screening, using Markov models and Monte Carlo simulation, respectively. ${ }^{12-14}$

The aim of this study was to develop a simple model to estimate the probable yield from a screening programme and its impact on the incidence of blindness in a diabetic population, 
based on contemporary evidence, and taking account of uncertainty about key variables, namely prevalence of treatable retinopathy, incidence of blindness in patients with retinopathy, sensitivity of screening tests, and therapeutic effectiveness of laser photocoagulation. The model is intended for adaptation and use by those responsible for purchasing and providing health care for geographically defined diabetic populations.

\section{Methods}

The study comprised a literature review with incorporation of relevant data into a quantitative model. The aim of the review was to obtain valid estimates for the following variables: prevalence of treatable retinopathy in diabetic populations; incidence of treatable retinopathy in diabetic patients; incidence of blindness and mortality in diabetic patients with treatable retinopathy; validity of screening tests in detecting treatable retinopathy; and effectiveness of treatment. Treatable retinopathy was defined as proliferative retinopathy, or nonproliferative retinopathy with macular oedema. Patients with these conditions have been shown to benefit from treatment in randomised controlled trials, as discussed below. Yield was defined as the percentage of screened patients correctly identified as having treatable retinopathy.

Three electronic bibliographic databasesMEDLINE, EMBASE, and Science Citation Index - were searched for the period January 1980 to June 1996. The mesh headings used for MEDLINE were "diabetic retinopathy" and "diagnosis", "incidence"; "prevalence" or "treatment"; "diabetes mellitus" and "vision screening", "prevalence", "laser" or "photoco-

Table 1 Inputs (research-based estimates) and outputs (consequences of screening and treatment) of the model

\begin{tabular}{|c|c|c|}
\hline & First screening round & $\begin{array}{l}\text { Annual screening } \\
\text { rounds* }\end{array}$ \\
\hline \multicolumn{3}{|l|}{ Input variables } \\
\hline $\begin{array}{l}\text { Number of people with diabetes screened in a } \\
\text { district-wide screening programme }\end{array}$ & 5000 & 5000 \\
\hline Prevalence of treatable retinopathy among screened & 5.5 & 1.9 \\
\hline Test sensitivity (\%) & 70 & 70 \\
\hline Test specificity (\%) & 95 & 95 \\
\hline Effectiveness of treatment (Relative risk) & 0.23 & 0.23 \\
\hline $\begin{array}{l}\text { Annual incidence of blindness without treatment in } \\
\text { patients with treatable disease }(\%)\end{array}$ & 7.5 & 7.5 \\
\hline $\begin{array}{l}\text { Annual mortality in patients with treatable disease } \\
(\%)\end{array}$ & 5.0 & 5.0 \\
\hline \multicolumn{3}{|l|}{ Consequences for a typical health district: ${ }^{\star}$} \\
\hline \multicolumn{3}{|l|}{ Results of screening tests } \\
\hline True positives & 193 & 67 \\
\hline False positives & 236 & 245 \\
\hline False negatives & 83 & 29 \\
\hline True negatives & 4489 & 4660 \\
\hline Referred to ophthalmologist (all positives) & 429 & 312 \\
\hline Positive predictive value (\%) & 45 & 21 \\
\hline Negative predictive value (\%) & 98.2 & 99.4 \\
\hline Likelihood ratio for positive screening test & 14.0 & 14.0 \\
\hline Likelihood ratio for negative screening test & 0.32 & 0.32 \\
\hline Yield $(\%)$ & 3.86 & 1.33 \\
\hline \multicolumn{3}{|l|}{ Results of treatment } \\
\hline Number treated & 193 & 67 \\
\hline $\begin{array}{l}\text { Number of cases of blindness prevented by } \\
\text { treatment within the first year after treatment }(\%\end{array}$ & & \\
\hline of treated; $\%$ of screened) & $11(5.7 ; 0.2)$ & $4(5.7 ; 0.1)$ \\
\hline $\begin{array}{l}\text { Cumulative number of cases of blindness } \\
\text { prevented by treatment within } 10 \text { years of } \\
\text { treatment (\% of treated; } \% \text { of screened) }\end{array}$ & $66(34.2 ; 1.3)$ & $23(34.2 ; 0.5)$ \\
\hline
\end{tabular}

${ }^{\star}$ From fifth round onwards (see text). ${ }^{\star \star}$ For different numbers screened, adjust proportionally.
KEY POINTS

- Although there has been no randomised controlled trial of diabetic retinopathy screening, the impact of a programme can be estimated.

- Critical factors for a programme's effectiveness are prevalence of treatable disease among screened patients, test validity, and treatment effectiveness.

- Efficiency may be increased by ensuring that high risk patients are screened, and by screening lowest risk patients less often.

agulation". For EMBASE, these terms as well as "diabetes mellitus retinopathy" and "health screening programme" were used as thesaurus terms. Additional papers citing identified papers on screening tests, laser photocoagulation trials and modelling were traced using Science Citation Index. References cited in papers identified in the searches also examined.

The quality of evidence provided by each study was appraised using criteria proposed by Sackett et al. ${ }^{15}$ Of studies of treatment effectiveness, only randomised controlled trials were included. Studies of prevalence and incidence were included if the population studied and, where relevant, if the sampling methods used were clearly described, and if valid diagnostic tests were used on all subjects. Prevalence and incidence studies confined to hospital based populations were excluded. Studies of diagnostic test validity were included if the population studied was defined, if the test was adequately described, and if the test was compared with a reference standard test comprising either retinal examination by an ophthalmologist or stereoscopic retinal photography. The first test is a pragmatic standard on which a decision to treat would often be based, and the second test provides the clearest view of the greatest part of the retina. ${ }^{5}$ Where two by two tables were provided or could be constructed from reported data, sensitivity estimates from the different studies were pooled, using statistical methods for cluster sample surveys. ${ }^{16}$ The diagnostic criteria used to calculate pooled sensitivity estimates were: retinopathy of grade 3 or above, preproliferative or proliferative retinopathy, and retinopathy requiring referral or further investigation or treatment. Ungradeable cases were excluded from calculations where possible.

Quantitative modelling was performed using Excel spreadsheets. Estimates for input variables were obtained from the literature review. The relations between these variables and the consequences of screening were expressed algebraically, as defined in the appendix. The yield from screening was assumed to be a function of the prevalence of treatable retinopathy in screened populations and the screening test sensitivity. The number of cases of blindness prevented was assumed to be a function of the number of cases treated, the expected incidence of blindness without treatment among cases with treatable retinopathy, the effective- 
ness of treatment, the death rate in people with treatable disease, and the number of years since treatment. The diabetic population was assumed to be in a steady state, with a constant distribution of risk factors for retinopathy, and with numbers of deaths in diabetic patients equal to numbers of new cases of diabetes.

Estimates for some input variables differed substantially between studies. Sensitivity analyses were performed to examine the degree of variation in model output estimates attributable to variations in retinopathy prevalence, blindness incidence, test sensitivity, and treatment effectiveness.

Simulation modelling was performed to assess the degree of random variation that would be expected in yield, numbers of cases referred to ophthalmologists, and numbers of cases treated. For each outcome 100 years were simulated using the random number generation tool in the Excel package and assuming Poisson distributions of independent random events.

\section{Results}

The findings of the literature review are presented first, followed by the estimates calculated from the model. The best estimates of key input variables are shown in table 1 and explained below. Plausible ranges of input variables are also discussed.

NUMBER OF PEOPLE WITH DIABETES SCREENED ANNUALLY

The average population per Family Health Service Authority or Health Board was 497000 in $1994 .{ }^{17}$ Four district wide studies

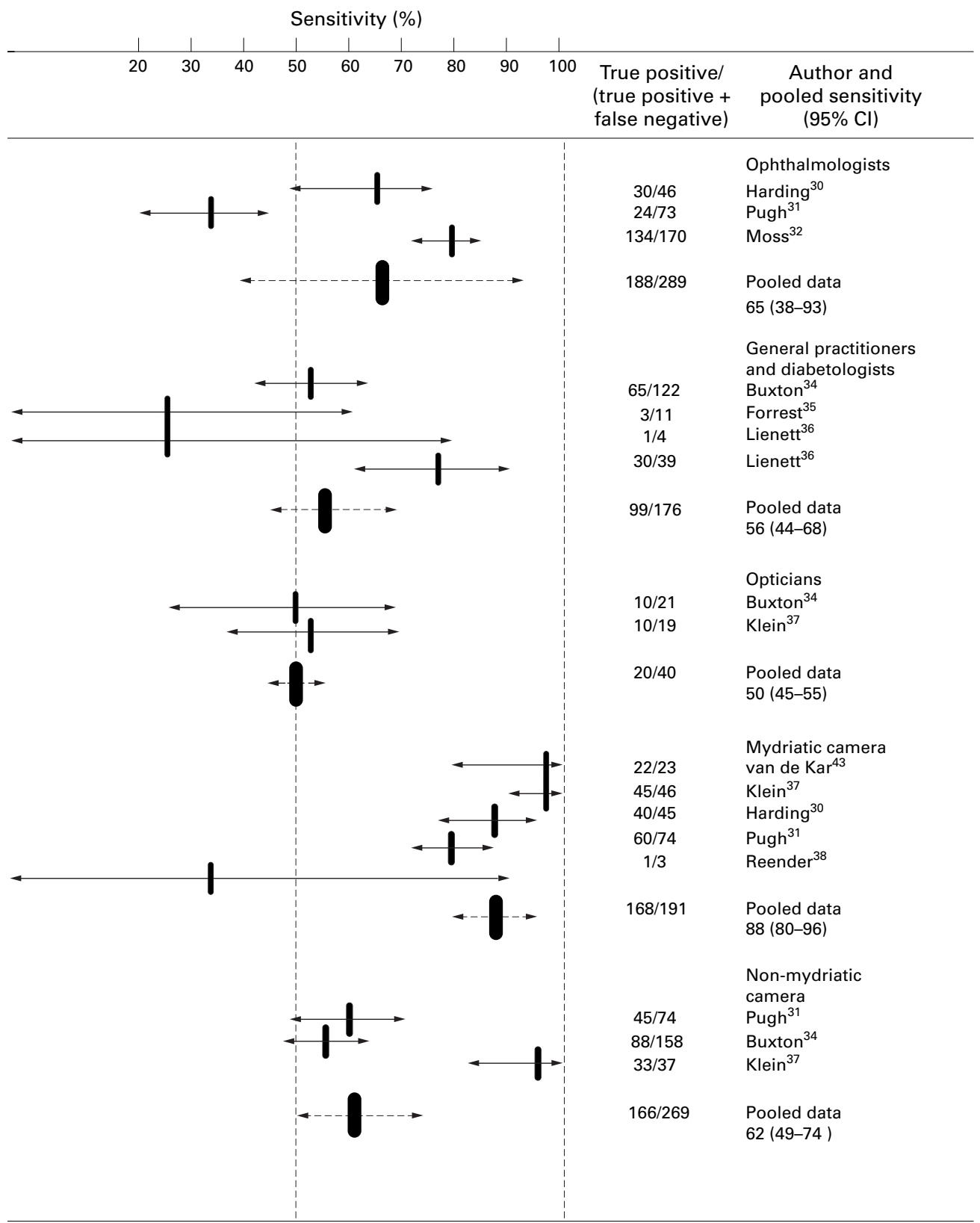

Figure 1 Sensitivity (point estimates and 95\% confidence intervals) of screening tests in diagnosing proliferative or sight threatening retinopathy, with either stereoscopic photography or ophthalmologist ophthalmoscopy as reference standard, for studies providing sufficient data, with pooled estimates from these studies. 


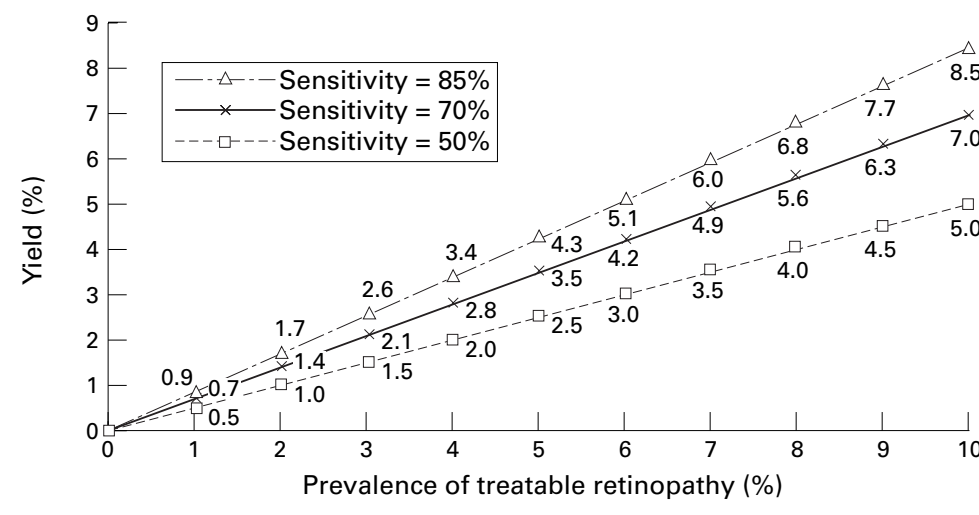

Figure 2 Sensitivity analysis of yield (treatable cases detected/number screened (\%)) for different estimates of test sensitivity and disease prevalence.

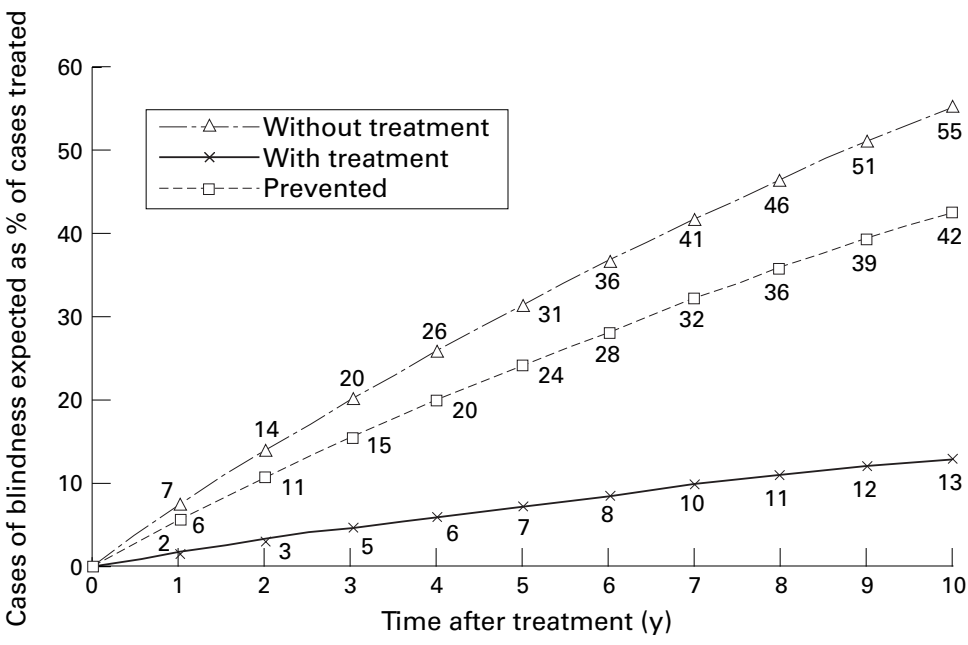

Figure 3 Cumulative cases of blindness expected with and without treatment, as percentage of patients with treatable disease who were diagnosed and treated.

estimated the prevalence of known diabetes among all patients registered with general practices to be $1.2 \%,{ }^{18} 1.2 \%,{ }^{19} 1.2 \%,{ }^{20}$ and $1.6 \%{ }^{21}$ respectively. Pooling data from the three studies that reported numerators and denominators $^{18} 2021$ provides a crude prevalence of diagnosed diabetes of $1.25 \%$. Thus for a district population of half a million about 6250 people would have diabetes. Populations with higher proportions of elderly or Asian populations would be expected to have higher prevalences of diabetes. ${ }^{22}$ If $80 \%$ of 6250 diabetic patients were screened annually, 5000 screening tests would be required.

PREVALENCE AND INCIDENCE OF TREATABLE RETINOPATHY IN DIABETIC PATIENTS

Two British studies examined previously unscreened general practice diabetic populations and found the prevalences of "referable retinopathy" 23 and of "sight threatening retinopathy" ${ }^{24}$ to be $5 \%$ and $6 \%$, respectively, using stereoscopic retinal photography or ophthalmologist ophthalmoscopy for diagnosis. Prevalence at an initial screening round would be likely to lie between these two estimates.

The Wisconsin cohort study of diabetic retinopathy ${ }^{25-29}$ reported incidences of proliferative retinopathy and clinically significant macular oedema, stratified by insulin use and age at onset of diabetes. To obtain an overall incidence rate, a weighted average of the stratum specific rates was calculated. In our experience in Avon in 1995, general practitioners reported that $26 \%$ of the 15000 diabetic patients known to them were being treated with insulin. Assuming that $26 \%$ of diabetic patients were insulin using, that of these, half first developed diabetes under 30 years of age, and that half of the cases of macular oedema occurred in patients who also developed proliferative retinopathy, an overall annual incidence of treatable retinopathy of $1.3 \%$ was obtained. This estimate is similar to the prevalence of sight threatening retinopathy $(1.5 \%)$ reported for the second screening round in the Oxford, Sheffield, and Exeter study. ${ }^{24}$

At successive screening rounds, prevalent cases would consist both of cases that arose since the last round, and cases missed at the last round. The prevalence of treatable retinopathy at successive screening rounds is thus a function of the incidence of retinopathy, the sensitivity of screening tests, and the prevalence at preceding rounds, as defined in the appendix. Assuming $70 \%$ test sensitivity and $1.3 \%$ annual incidence, the prevalence at successive screening rounds would be $5.5 \%$, $3.0 \%, 2.2 \%, 2.0 \%$, and would reach a constant prevalence of $1.9 \%$ from the fifth round on.

\section{SENSITIVITY AND SPECIFICITY OF SCREENING} TESTS

Figure 1 shows that reported test sensitivities varied widely. Pooled data suggest that screening by mydriatic camera was most sensitive $(88 \%)$, and that other tests did not significantly differ from each other (point estimates from $50 \%$ to $65 \%$ with overlapping confidence intervals). O'Hare et $a l^{23}$ reported sensitivities of screening by optician (73\%), general practitioner $(56 \%)$, non-mydriatic retinal camera $(71 \%)$, and various combinations of tests $(60 \%-88 \%)$ but provided insufficient detail for inclusion in the pooled estimates. The relatively high sensitivities in the latter study may be because a lower diagnostic threshold was used for referable retinopathy than was used for treatable retinopathy in the other studies. For the purposes of the model it was assumed that a screening programme with thorough training and quality control could feasibly attain a sensitivity of $70 \%$. Published specificity estimates did not vary widely, and were usually between $90 \%$ and $100 \% .^{30-38}$ A specificity of $95 \%$ was assumed for the model.

EFFECTIVENESS OF TREATMENT

Laser photocoagulation can arrest the progression of proliferative retinopathy and macular oedema and prevent severe visual loss and blindness. A meta-analysis of five randomised controlled trials with between 1.5 and five years follow up was performed by Rohan et al. ${ }^{11}$ Compared with no treatment, the relative risk of severe visual loss or blindness after treatment, in eyes with proliferative retinopathy or macular oedema was 0.39 (95\% CI 0.28 , 0.55). A subsequent randomised controlled 
trial $^{39-41}$ found that treatment at earlier stages of retinopathy was less effective. For eyes with non-proliferative or early proliferative retinopathy laser photocoagulation had a relative risk over five years of 0.77 (99\% CI $0.56,1.06$ ) compared with deferral of photocoagulation until retinopathy progressed to more severe grades. For the subgroup of patients with both macular oedema and mild to moderate nonproliferative retinopathy, treatment was more effective (relative risk $=0.44$ ), which is in keeping with the findings of the five earlier trials. Because the trials used eyes and not people as units of randomisation, the magnitude of effect of treatment in preventing blindness in people could not be assessed. If treatment of one eye is more effective than treatment of the other eye, the prevention of visual loss will be determined by the effectiveness of treatment in the eye with the best vision. Rohan et al estimated that if effectiveness in two eyes was totally independent, then the relative risk of blindness in treated people compared with untreated people would be 0.15 (0.39 $\times$ 0.39). ${ }^{11}$ As effectiveness in both eyes is unlikely to be completely independent, the relative risk of blindness in a person would lie between 0.15 and 0.39 , with a midpoint estimate of $0.23 .^{11}$

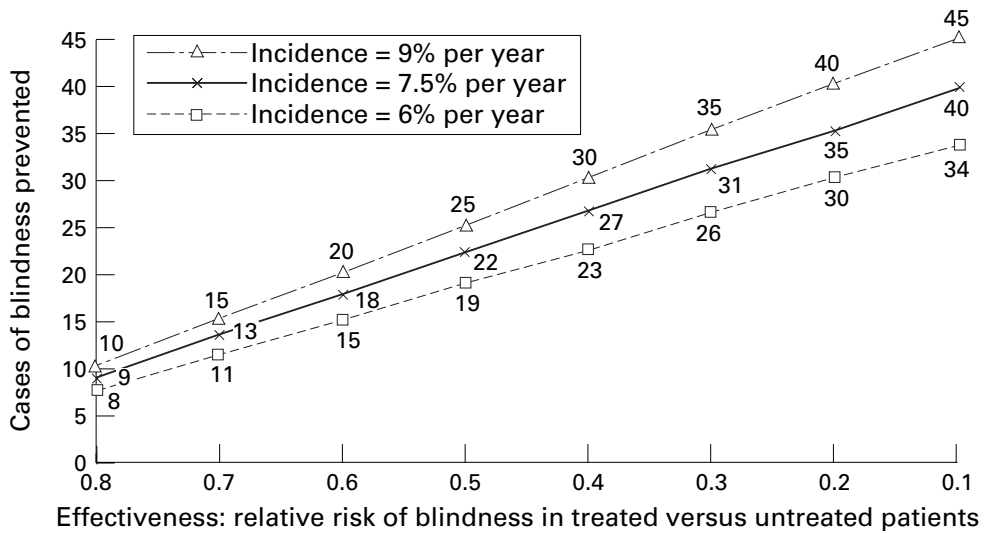

Figure 4 Sensitivity analysis: cumulative number of cases of blindness prevented over 10 years if 100 patients requiring treatment were treated, for varying estimates of blindness incidence without treatment and therapeutic effectiveness.

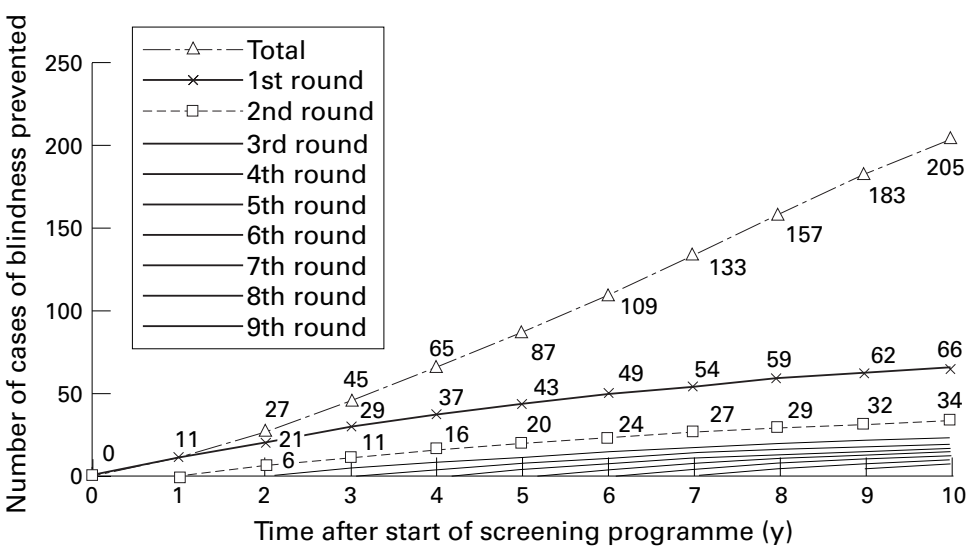

Figure 5 Cumulative cases of blindness prevented for a screening programme examining 5000 diabetic patients and repeated over 10 consecutive years.
INCIDENCE OF BLINDNESS AND MORTALITY IN DIABETIC PATIENTS WITH TREATABLE RETINOPATHY

In the Wisconsin cohort study, $24 \%$ of right eyes with proliferative or severe nonproliferative retinopathy at baseline became blind (acuity $<20 / 200$ ) within four years. ${ }^{27}$ Thus, assuming a constant incidence over four years, the annual incidence was about $6 \%$. Incidence in left eyes was similar. In the Diabetic Retinopathy Study trial of laser photocoagulation, $27 \%$ of untreated eyes developed severe visual loss (acuity < 5/200) over three years, thus the annual incidence was about $9 \% .^{42}$ For the purposes of the model the annual incidence of blindness in untreated eyes with treatable retinopathy was estimated to be $7.5 \%$, which is midpoint between $6 \%$ and $9 \%$. In the Wisconsin cohort study, patients with proliferative retinopathy had an annual death rate of $5 \% .^{29}$

\section{MODEL RESULTS}

Cases of treatable retinopathy detected

During an initial screening round in a previously unscreened population of diabetic patients registered with general practitioners, about $3.9 \%$ would be correctly diagnosed as having treatable retinopathy (table 1). Yield would decrease to $2.1 \%, 1.5 \%, 1.4 \%$, and $1.3 \%$ in the second, third, fourth, and fifth rounds, respectively, and stabilise at $1.3 \%$ thereafter. The sensitivity analysis (fig 2) shows the range of yields that could be obtained with different test sensitivities and prevalences of treatable retinopathy. The positive predictive value represents the proportion of patients with positive screening test results who required treatment. This would decrease from $45 \%$ in the first screening round to $21 \%$ in annual rounds (table 1 ). The negative predictive value is the probability of not having treatable retinopathy if the screening test is negative. This would increase very slightly in subsequent rounds (table 1). Increasing test sensitivity to $85 \%$ would increase yield to $4.7 \%$ in an initial screening round and would stabilise at $1.3 \%$ in annual rounds.

The number of treatable cases detected in the population of a general practice would be expected to vary widely because of small numbers. A practice with a list size of 10000 would be expected to screen 100 patients per year. Simulation modelling predicts that in the first year of screening there would be a $95 \%$ probability of detecting at least one case but in annual rounds there would be a $50 \%$ probability of detecting at least one case.

Variation in workload for an ophthalmology department assessing all screening test positive cases, and treating all true positive cases, was also simulated. In an initial screening round covering 5000 patients there would be a $90 \%$ probability that between 393 and 463 cases would be referred and that between 172 and 209 cases would require treatment. After subsequent screening rounds there is a $90 \%$ probability that between 288 and 343 cases would be referred and between 55 and 76 cases would require treatment. Thus the relative 
magnitude of random variation in annual requirements for laser photocoagulation would increase as the prevalence of treatable disease decreased.

\section{Cases of blindness prevented}

The increasing numbers of cases of blindness prevented in the years after a single round of screening and treatment are shown in figure 3. Of patients treated, almost $6 \%$ would be prevented from going blind within a year, and $34 \%$ within 10 years (table 1 ). These numbers are equivalent to $0.2 \%$ and $1.6 \%$ of all patients screened in the first round, and $0.1 \%$ and $0.6 \%$ of all patients screened in annual rounds (table 1). Figure 4 shows the sensitivity of the model to assumptions about the incidence of blindness without treatment and the effectiveness of treatment. Even if treatment was much less effective than is reported in the literature, substantial proportions of treated patients would benefit.

Figure 5 shows the cumulative number of cases of blindness prevented by a screening programme repeated over 10 consecutive years, and the numbers consequent on each year's screening. The first year of screening and treatment contributes more to prevention than do subsequent years, because of the declining yield. The total number of cases of blindness prevented would however continue to increase steadily, both because of additional cases treated and because of the cumulative benefits over time of each treatment round.

\section{Discussion}

The literature review and model show that screening and treatment can prevent many cases of blindness caused by diabetic retinopathy but that the proportion of screened patients who would benefit from treatment in the short-term is small. The effectiveness of screening could be increased by increasing test sensitivity and by improving uptake among diabetic patients at greatest risk of retinopathy. Mydriatic retinal photography is consistently the most sensitive test, and its wider use would be likely to increase screening effectiveness, but other tests have the potential to perform well. In areas with well established screening by ophthalmoscopy it may be more appropriate to improve test performance by continuing training of examiners and audit than to abandon ophthalmoscopy for retinal photography. Repeated screening would help detect cases missed initially.

A particular effort should be made to encourage regular examination of high risk patients, that is those with diabetes of long duration, and patients found to have non-proliferative retinopathy at previous examinations, by means of practice or district based registers and invitations to attend. The percentage yield could also be increased by excluding, or introducing longer screening intervals for, diabetic subgroups at low risk of treatable retinopathy. In the Wisconsin cohort study less than $0.4 \%$ of patients with no evidence of retinopathy at baseline had proliferative retinopathy four years later. ${ }^{25}$ The yield obtained by screening patients a year after finding no retinopathy would thus be less than one per thousand. Two problems of implementing longer screening intervals for patients with negative test results are that treatable cases may be missed, and that variable screening intervals would be more confusing for patients and difficult to administer. It is has become conventional for all people with diabetes to have annual review examinations, ${ }^{23}$ and it may be simpler and less costly to include retinopathy screening as part of the annual review than to provide it separately. ${ }^{24}$ Yield could also be increased by excluding low risk patients such as those with diabetes of less than five years duration. ${ }^{4}$

The difference between treatable and referable retinopathy is ambiguous. The first, more restrictive, category has been adopted for this model because it is more relevant in estimating blindness prevented through treatment. If the threshold for referral was lowered by lowering the diagnostic threshold of the test to include less severe grades of retinopathy, fewer cases would be missed and more blindness would be prevented. However, the accompanying fall in specificity would lead to more referrals of false positives and increased pressure on ophthalmological resources. Methods for metaanalysing trade offs between sensitivity and specificity in diagnostic tests by examining summary receiver operating characteristics have been described ${ }^{44}$ but were beyond the scope of this study.

Although the estimates of retinopathy prevalence used in this study were derived from general practice based diabetic populations, the model can be applied to hospital patients by adjusting the prevalence, incidence, and mortality estimates.

A recent description of screening programmes operating in 12 British centres, for an average of four years each, reported that $1.2 \%$ of screened patients, and $21.5 \%$ of referred patients, received laser therapy. ${ }^{6}$ These figures are remarkably similar to this model's estimated yield (1.3\%) and positive predictive value $(21 \%)$ for annual screening rounds, supporting the validity of the model. In districts without coordinated screening programmes at present, it is probable that variable proportions of diabetic patients are being screened anyway, thus the notion of an initial prevalence round is somewhat hypothetical. If universal screening programmes were introduced, the initial consequences would be expected to lie somewhere between those shown in the two columns of table 1 . The validity of the model's estimates of blindness prevention is more difficult to assess. It is plausible that cases with milder forms of retinopathy than those defined as treatable in the model might in practice be treated. If so, then more cases may benefit, but the additional cases would be less likely to be effectively treated. Thus the absolute benefit would be higher but with decreasing marginal returns.

The economic dimensions of screening were beyond the scope of this study. A leading problem with published cost effectiveness analyses comparing different screening tests is the wide variation in test sensitivities reported, therefore no single test is clearly the most cost effective. ${ }^{5}$ United States cost-benefit analyses have sug- 
gested that retinopathy screening results in net cost savings, mainly through reduced social security payments for blindness, ${ }^{12-14}$ but these results are not readily generalisable to other countries. While much of the cost of blindness is borne by social security agencies, costs of screening and treatment are borne by health authorities.

Quantitative modelling is a logical and useful way of structuring the analysis of a complex issue, and for synthesising a range of published evidence. Local data can be incorporated into the model and updated as appropriate. A more sophisticated model could analyse different risk strata separately and simulate changes from year to year, ${ }^{12-14}$ but this would be more complex to reproduce using local data. Priorities for further research include controlled evaluations of attempts to improve screening test performance in field settings ${ }^{45}$ and to increase screening uptake among people with diabetes at highest risk of retinopathy, ${ }^{46}$ and comparative monitoring of yield ${ }^{6}$ and blindness incidence in comparable populations using different screening methods.

The authors are grateful to John Sparrow, who suggested modelling and provided references, to Chris Hine, who provided a purchaser perspective, and to Roy Taylor who commented on an earlier version of the manuscript. Responsibility for the paper lies with the authors.

Funding: none

Conflicts of interest: none

\section{Appendix: Formulas used to calculate prevalence at successive screening rounds, yield, and blindness prevented}

(1) Prevalence of treatable retinopathy at successive screening rounds $=$ annual incidence of treatable retinopathy+ $((1-$ sensitivity $) \times$ prevalence at previous screening round).

(2) Screening test results

Yield $=$ prevalence $\times$ sensitivity

True positives $=$ sensitivity $\times$ prevalence $\times$ number screened

False

negatives $=(1-$ sensitivity $) \times$ prevalence $\times$ number screened

True negatives $=$ specificity $\times(1-$ prevalence $) \times$ number screened

False positives $=(1-$ specificity $) \times(1-$ prevalence $) \times$ number screened

Positive predictive value $=$ true positives $/$ (true positives + false positives)

Negative predictive value $=$ true negatives $/$ (true negatives + false negatives)

Likelihood ratio for positive screening test= true positives/(true positives + false negatives) /false positives $\times$ (false positives + true negatives)

Likelihood ratio for negative screening test $=$ false negatives/(true positives +false negatives)/ true negatives $\times$ (true negatives + false positives)

(3) Cases of blindness prevented

Number of cases of blindness prevented during a year=number of cases at risk at the start of the yearxexpected annual incidence of blindness without treatment $\times(1-$ relative risk in treated versus untreated)

Number of detected cases of treatable disease at risk of blindness at start of first year
$=$ number of true positive cases detected by screening

Number at risk of blindness without treatment at start of $n$th year after treatment= number at risk at start of previous year $\times(1-$ death rate-annual incidence of blindness without treatment $)=R_{n}$

Number of cases of blindness prevented during the $n$th year $=R_{n} \times$ annual incidence of blindness without treatment $\times(1 \times$ relative risk in treated versus untreated)

Cumulative number of cases of blindness prevented to year $k$ after treatment=sum of cases of blindness prevented each year from year 1 to year $k$

1 Williams R. Diabetes mellitus. In: Stevens A, Raftery J, eds. Health care needs assessment. Oxford: Oxford University Press, 1994: 31-57.

2 British Diabetic Association. Guidelines on the management of diabetes in primary care. London: British Diabetic Association, 1994 .

3 Clinical Standards Advisory Group. Standards of clinical care for people with diabetes. London: HMSO, 1994.

4 Singer DE, Nathan DM, Fogel HA, Schachat AP. Screening for diabetic retinopathy. Ann Intern Med 1992;116:660-71.

5 Mason J, Drummond M. Screening for diabetic retinopathy by optometrists: effectiveness and cost-effectiveness. Centre for optometrists: effectiveness and cost-effectiveness. Centre for of York, 1995 .

6 Taylor R, British Diabetic Association Mobile Retinal Screening Group. Practical community screening for diabetic retinopathy using the mobile retinal camera: report of a 12 centre study. Diabet Med 1996;13:946-52.

7 Taylor R, Lovelock L, Tunbridge WMG, et al. Comparison

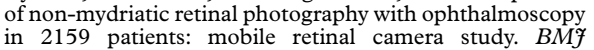
1990;301:1243-7.

8 Leese GP, Ahmed S, Newton RW, et al. Use of mobile screening unit for diabetic retinopathy in rural and urban areas. BMF 1993;306:187-9.

9 Kristinsson JK, Stefansson E, Jonasson F, et al. Systematic screening for diabetic eye disease in insulin dependent diabetes. Acta Ophthalmol 1994;72:72-8.

10 Kristinsson JK, Stefansson E, Jonasson F, et al. Systematic screening for diabetic eye disease in type 2 diabetes screening for diabetic eye disease in
mellitus. Acta Ophthalmol 1994;72:341-6.

11 Rohan TE, Frost CD, Wald NJ. Prevention of blindness by screening for diabetic retinopathy: a quantitative assessment. BMF 1989;299:1198-201.

12 Dasbach EJ, Fryback DG, Newcomb PA, et al. Costeffectiveness of strategies for detecting diabetic retinopathy. Med Care 1991;29:20-39.

13 Javitt JC, Canner JK, Frank RG, et al. Detecting and treating retinopathy in patients with type I diabetes mellitus. A health policy model. Ophthalmology 1990;97:483-95.

14 Javitt JC, Canner JK, SommerA. Cost effectiveness of current approaches to the control of retinopathy in type 1 diabetes mellitus. Ophthalmology 1989;96:255-64.

15 Sackett DL, Haynes RB, Guyatt GH, et al. Clinical epidemiology. A basic science for clinical medicine. Boston: Little, ology. A basic sc

16 Bennett S, Woods T, Liyanage WM, et al. A simplified general method for cluster-sample surveys of health in developing countries. World Health Stat Q 1991;44:98-106.

17 Compendium of Health Statistics. London: Office of Health Economics, 1995.

18 Howitt AJ, Cheales NA. Diabetes registers: A grassroots approach. BMf 1993;307:1046-8.

19 Benett IJ, Lambert C, Hinds G, et al. Emerging standards for diabetes care from a city-wide primary care audit. Diabet Med 1994;11:489-92.

20 Burnett SD, Woolf CM, Yudkin JS. Developing a district diabetic register. $B M \mathcal{F}$ 1992;305:627-30.

21 Goulder J, Balme M, Hooper P. Structuring diabetes care within general practice Practical Diabetes 1993;10:178-81.

22 Simmons D, Williams DRR, Powell MJ. The Coventry Diabetes Study: Prevalence of diabetes and impaired glucose tolerance in Europids and Asians. Q $\mathcal{F}$ Med 1991;81:102130

23 O'Hare JP, Hopper A, Madhaven C, et al. Does retinal photography improve screening for diabetic retinpathy in the primary care setting? $B M \mathcal{F} 1996 ; \mathbf{3 1 2}: 678-82$.

24 Sculpher MJ, Buxton MJ, Ferguson BA, et al. Screening for diabetic retinopathy: a relative cost-effectiveness analysis of alternative modalities and strategies. Health Econ 1992;1: 39-51.

25 Klein R, Klein EK, Moss SE, et al. The Wisconsin epidemiologic study of diabetic retinopathy. Arch Opthalmol 1989;107:237-49.

26 Klein R, Klein BEK, Moss SE. The Wisconsin epidemiologic study of diabetic retinopathy: an update. Aust $N Z \mathcal{F}$ Ophthalmol 1990;18:19-21.

27 Moss SE, Klein R, Klein BEK. The incidence of vision loss in a diabetic population. Ophthalmology 1988; 95:1340-8. 
28 Klein R, Klein BEK, Moss SE, et al. The Wisconsin Epidemiologic Study of Diabetic Retinopathy. IV. Four year incidence and progression of diabetic retinopathy when age at 237-43.

29 Klein R, Moss SE, Klein BEK, et al. Relation of ocular and systemic factors to survival in diabetes. Arch Intern Med 1989;149:266-72

30 Harding SP, Broadbent, Neoh C, et al J. Sensitivity and specificity of photography and direct ophthalmoscopy in screening for sight threatening eye disease: the Liverpoo diabetic eye study. BMF 1995;311:1131-5.

31 Pugh JA, Jacobson JM, Van Heuven WA, et al. Screening for diabetic retinopathy. The wide angle retinal camera. Diabetes Care 1993;16:889-95.

32 Moss SE, Klein R, Kessler SD, et al. Comparison between ophthalmoscopy and fundus photography in determining severity of diabetic retinopathy. Ophthalmology 1985;92: s2-7.

33 Williams R, Nussey S, Humphry R, et al. Assessment of non-mydriatic fundus photography in detection of diabetic non-mydriatic fundus photography in
retinopathy. $B M \mathcal{F}$ 1986;293:1140-2.

34 Buxton MJ, Sculpher MJ, Ferguson BA, et al. Screening for treatable diabetic retinopathy: a comparison of differen Diabet Med 1991;8:371-7.

35 Forrest RD, Jackson CA, Yudkin JS. Screening for diabetic retinopathy - comparison of a nurse and a doctor with retinal photography. Diabetes Res 1987;5:39-42.

36 Lienett RT. Inter-observer comparisons of ophthalmoscopic assessment of diabetic retinopathy. Aust $\mathrm{NZ}$ F Ophthalmol 1989;17:363-8.

37 Klein R, Klein BEK, Neider MW, et al. Diabetic retinopathy as detected using ophthalmoscopy, a nonmydriatic camera and a standard fundus camera. Ophthalmology 1985;92 485-91.
38 Reenders K, de Noble E, van den Hoogen $\mathrm{H}$, et al. Screening for diabetic retinopathy by general pracitioners. Scand $\mathcal{F}$ Prim Health Care 1992;10:306-9.

39 Early Treatment Diabetic Retinopathy Research Group. Photocoagulation for diabetic macular edema. Arch Ophthalmol 1985;103:1796-806.

40 Early Treatment Diabetic Retinopathy Research Group. Early Treatment Diabetic Retinopathy Study design and baseline patient characteristics. EDTRS report number 8. Ophthalmology 1991;98:741-56.

41 Early Treatment Diabetic Retinopathy Research Group. Early photocoagulation for diabetic retinopathy. EDTRS report number 9. Ophthalmology 1991;98:766-85.

42 Diabetic Retinopathy Study Research Group. Photocoagulation treatment of proliferative diabetic retininopathy. Clinical application of Diabetic Retinopathy Study (DRS) findings. DRS report number 8. Ophthalmology 1981;88: 583-600.

43 van de Kar W, van der Velden HG, van Weel C, et al. Diagnosing retinopathy by general practitioners and by a hospial physician. The use of fundus photos. Scand $\mathcal{F}$ Prim Health Care 1990;8:19-23.

44 Irwig L, Macaskill P, Glasziou P, et al. Meta-analytic methods for diagnostic accuracy. $\mathcal{F}$ Clin Epidemiol 1995;48:11930 .

45 Awh CC, Cupples HP, Javitt JC. Improved detection and referral of patients with diabetic retinopathy by primary care physicians. Effectiveness of education. Arch Intern Med 1991;151:1405-8.

46 Hurwitz B, Goodman C, Yudkin J. Prompting the clinical care of non-insulin-dependent (type-II) diabetic-patients in an inner-city area - one model of community care. $B M \mathcal{F}$ 1993;306:624-30. 